\title{
Result of an endoscopic mucosal resection demonstrated at the International Gastric Cancer Congress in New York
}

\author{
Takuji Gotoda ${ }^{1}$, Hitoshi Kondo ${ }^{1,2}$, Hiroyuki Ono ${ }^{1}$, Daizo Saito ${ }^{1}$, and Tadakazu Shimoda ${ }^{3}$ \\ ${ }^{1}$ Endoscopy Division, National Cancer Center Hospital, 5-1-1, Tsukiji, Chuo-Ku, Tokyo 104-0045, Japan \\ ${ }^{2}$ The Center for Digestive Disease, Tonan Hospital, Sapporo, Japan \\ ${ }^{3}$ Clinical Laboratory Division, National Cancer Center Hospital, Tokyo, Japan
}

To the Editor:

At the opening ceremony of the fourth International Gastric Cancer Congress, held in New York in May 2001, we had the opportunity to demonstrate, via satellite transmission live from Tokyo, a new procedure of endoscopic mucosal resection (EMR) for early gastric cancer. Fortunately, and to the great relief of our colleagues anxiously watching the platform screen in New York, the EMR went smoothly and the resected specimen was retrieved from the patient's stomach in full view of the large audience.

We believe it is our obligation to report the histological findings of the specimen and the subsequent clinical course of the patient.

The patient, a 69-year-old man, was admitted on the day of the demonstration. The lesion was a $2-\mathrm{cm}$ reddish depression on the anterior wall of the lower gastric body (Fig. 1). The biopsy revealed well-differentiated adenocarcinoma, and the depth of invasion was endoscopically estimated as $\mathrm{T} 1$ limited to the mucosal layer. Based on these findings, EMR was determined to be the best therapeutic option for him, as the risk of lymph node metastasis was low [1].

After indigocarmine spray marking was done to contrast the minute mucosal structure, we performed EMR, using an insulation-tipped electrosurgical (IT) knife [2] (Figs. 2-9). The procedure between the marking (Fig. 2) and resection (Fig. 8) took $15 \mathrm{~min}$. Endoscopy on the following day confirmed no bleeding or perforation. The patient fasted for $48 \mathrm{~h}$ and was discharged on the fifth day without any complication. Recently we performed endoscopy for his 12-month follow-up that revealed only an ulcer scar.

Histological examination of the resected specimen showed that the tumor was confined to the mucosal layer without lymphatic or vascular capillary involvement (Figs. 10, 11). The resection lines, both vertical and lateral, were free of cancer invasion. The resection was thus considered to be curative both clinically and histologically.

EMR has become an important treatment option for early gastric cancer in Japan [3]. The gastric cancer treatment guidelines issued by the Japanese Gastric Cancer Association (JGCA) in 2001 clearly defined the criteria for EMR [4]. The endoscopic technique varies among different institutions, and our IT knife method is suitable for the resection of large tumors or those with an ulcerative component. The JGCA criteria for EMR are quite strict, and the possibility of their expansion is a current topic among Japanese endoscopists. We believe that more patients with gastric cancer will benefit from this noninvasive method in the future.

\section{References}

1. Gotoda T, Yanagisawa A, Sasako M, Ono H, Nakanishi Y, Shimoda $\mathrm{T}$, et al. Incidence of lymph node metastasis from early gastric cancer: estimation with a large number of cases at two large centers. Gastric Cancer 2000;3:219-25.

2. Gotoda T, Kondo H, Ono H, Saito Y, Yamaguchi H, Saito D, et al. A new endoscopic mucosal resection (EMR) procedure using an insulation-tipped diathermic (IT) knife for rectal flat lesions. Gastrointest Endosc 1999;50:560-3.

3. Sano T, Katai H, Sasako M, Maruyama K. The management of early gastric cancer. Surg Oncol 2000;9:17-22.

4. Nakajima T. Gastric cancer treatment guidelines in Japan. Gastric Cancer 2002;5:1-5. 

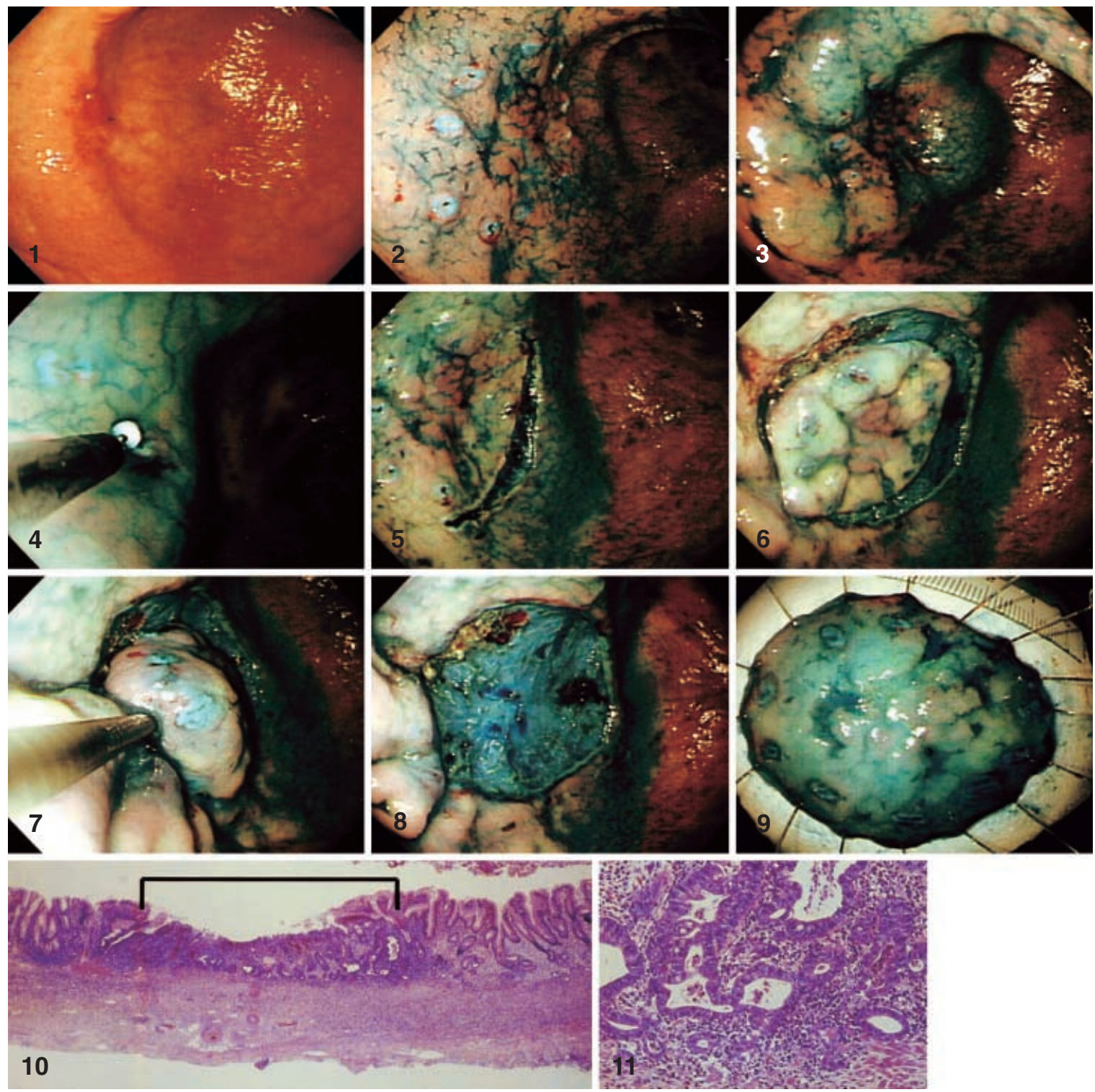

Fig. 1. Reddish depression on the anterior wall of the lower gastric body

Fig. 2. Marking dots on the circumference of the lesion after indigocarmine spraying

Fig. 3. Injection of saline with epinephrine $(0.025 \mathrm{mg} / \mathrm{ml})$ into the submucosal layer around the lesion to lift it off the muscle layer

Fig. 4. Insertion of the tip of the insulation-tipped electrosurgical (IT) knife into the submucosal layer through the initial incision

Fig. 5. Using END-CUT mode (ERBE ICC 200, Germany)

Fig. 6. Complete separation from the surrounding nonneoplastic mucosa

Fig. 7. Resection using standard snare polypectomy

Fig. 8. Complete resection without major complications

Fig. 9. The resected material, which is $45 \mathrm{~mm}$ in diameter pinned on a flat cork

Fig. 10. Depressed (IIc) type intramucosal cancer that is $20 \mathrm{~mm}$ in size. $(\mathrm{H} \& \mathrm{E}, \times 4)$

Fig. 11. Well-differentiated adenocarcinoma with high-grade atypia. $(\mathrm{H} \& \mathrm{E}, \times 40)$ 